\title{
1 Efficient multiplex genome editing using CRISPR-Mb3Cas12a in mice
}

3 Zhuqing Wang ${ }^{1,5}$, Yue Wang ${ }^{1,5}$, Shawn Wang ${ }^{1}$, Andrew J Gorzalski ${ }^{2}$, Hayden

4 McSwiggin ${ }^{1}$, Tian $\mathrm{Yu}^{1}$, Kimberly Castaneda-Garcia ${ }^{1}$, Huili Zheng ${ }^{1}$, and Wei Yan ${ }^{1,3,4, *}$

$6{ }^{1}$ Department of Physiology and Cell Biology, University of Nevada, Reno School of

7 Medicine, Reno, NV 89557.

$8 \quad{ }^{2}$ Nevada State Public Health Laboratory, University of Nevada, Reno School of

9 Medicine, Reno, NV 89557.

$10{ }^{3}$ Department of Obstetrics and Gynecology, University of Nevada, Reno School of

11 Medicine, Reno, NV 89557.

$12{ }^{4}$ Department of Biology, University of Nevada, Reno, Reno, NV 89557.

$13{ }^{5}$ These authors contributed equally to this work.

15 Keywords: Genome editing, CRISPR, Cas9, Cas12a, Cpf1, PAM

${ }^{*}$ Corresponding author:

Wei Yan M.D., Ph.D.

21 University of Nevada, Reno Foundation Professor

22 Department of Physiology and Cell Biology

23 University of Nevada School of Medicine

24 Center for Molecular Medicine, Room 207B

251664 North Virginia Street, MS/0575

26 Reno, NV 89557

27 Tel: 7757847765

28 Fax: 7757844362

29 Email: wyan@med.unr.edu 


\section{Summary statement}

2 CRISPR-Mb3Cas12a can target a broader range of sequences in murine zygotes

3 compared to AsCas12a and LbCas12a, and has lower on-target effects than Cas9 and

4 high overall knock-in efficiency.

5

6 Abstract

7 Despite many advantages over Cas9, Cas12a has not been widely used in genome

8 editing in mammalian cells largely due to its strict requirement of the TTTV protospacer

9 adjacent motif (PAM) sequence. Here, we report that Mb3Cas12a (Moraxella bovoculi

10 AAX11_00205) could edit the genome in murine zygotes independent of TTTV PAM

11 sequences and with minimal on-target mutations and close to $100 \%$ editing efficiency

12 when crRNAs of 23nt spacers were used.

13 


\section{Introduction}

2 The rapid advancement of CRISPR-Cas-based genome editing technologies has made gene therapy increasingly promising. However, several obstacles remain, including safety concerns due to both off-target and on-target mutations (Adikusuma et al., 2018;

5 Fu et al., 2013; Hsu et al., 2013; Kosicki et al., 2018; Lee and Kim, 2018) and the requirement of proper PAM sequences for efficient and precise cleavage by the commonly used endonucleases (Komor et al., 2017), e.g., Cas9 and Cas12a/Cpf1. The

8 Cas12a endonuclease has several advantages over Cas9. First, the most commonly used SpCas9 requires NGG PAM sequence, whereas the other widely used AsCas12a and LbCas12a utilize TTTV PAM sequences for efficient genome editing (Cong et al., 2013; Jinek et al., 2012; Wang et al., 2013; Zetsche et al., 2015). Second, Cas12a is guided by a single short CRISPR RNA (crRNA) and can efficiently process its own crRNAs, while Cas9 is directed by dual RNAs consisting of a crRNA and a tracrRNA, and rarely processes its own crRNAs (Cong et al., 2013; Fonfara et al., 2016; Jinek et al., 2012; Zetsche et al., 2017a). Third, Cas12a has much reduced off-target effects compared to SpCas9 due to its irreversible binding to the target region and strong discrimination against the off-target sequences (Kim et al., 2017; Kleinstiver et al., 2016; Strohkendl et al., 2018). Finally, Cas9 has been shown to cause on-target mutations including large deletions and insertions (Adikusuma et al., 2018; Kosicki et al., 2018; Lee and Kim, 2018), whereas Cas12a only generates staggered DNA overhangs, which may lead to much lower rate of on-target mutations due to the so-called preferred microhomology-mediated end joining (MMEJ) repair mechanism (Zetsche et al., 2015). However, practical applications of Cas12a have been severely hindered due, at least in

24 part, to its strict requirement for the TTTV PAM sequence. Although the PAM sequence for FnCas12a has been shown to be YTV ( $Y$ stands for $C / T$ and $V$ for $A / C / G$ ), the actual editing efficiency of YTV PAM sequences in mammalian cells remains rather low (Tu et al., 2017; Zetsche et al., 2015). Inspired by a recent report suggesting that Mb3Cas12a edits HEK293 cells at a much higher efficiency through TTV PAM sequences compared to AsCas12a and LbCas12a (Zetsche et al., 2017b preprint), we explored whether Mb3Cas12a could be utilized for efficient genome editing and production of knockout/knock-in mice lines. 
2 Results and Discussion

3 To determine whether Mb3Cas12a is active in mouse zygotes, we first used one crRNA

4 harboring 20nt direct repeats with 20nt spacer recognizing the TTTV PAM sequence of

$5 \quad$ Prps1/1 (Table 1, Supplementary Fig. 1A, D), a testis-specific gene dispensable for

6 spermatogenesis (Wang et al., 2018). One out of six founders obtained has an indel

7 (insertion or deletion) (16.7\%) (Table 1, Supplementary Fig. 1A, D), suggesting that

8 Mb3Cas12a indeed works in mouse zygotes. Since AsCas12a and LbCas12a have the

9 ability to process their own crRNAs (Fonfara et al., 2016; Zetsche et al., 2017a), we

10 next tested whether Mb3Cas12a could do the same. We designed one crRNA

11 harboring two 20nt spacers recognizing two TTTV PAM sequences at Saraf locus

12 (Table 1, Supplementary Fig. 1B, E). The 20nt spacers were separated by 20nt direct

13 repeats. One out of three founders was edited by spacer 1 (33.3\%) (Table 1 ,

14 Supplementary Fig. 1B, E), suggesting that Mb3Cas12a can indeed process its own crRNAs to edit a specific locus in mouse zygotes. We then determined whether Mb3Cas12a could utilize the TTV PAM sequence in mouse zygotes. We designed one crRNA harboring two 20nt spacers recognizing two TTV PAM sequences of the same Saraf locus (Table 1, Supplementary Fig. 1B, E). One out of four founders was edited with the spacer 2 (25\%) (Table 1, Supplementary Fig. 1B, E), indicating that Mb3Cas12a can indeed target genomic DNA with TTV PAM sequences. Since the

21 orientation of crRNAs has no significant effect on genome editing efficiency (Zetsche et

22 al., 2017a), we compared the efficiency of Mb3Cas12a in editing DNA harboring TTTV

23 and TTV PAM sequences by utilizing one single crRNA containing two spacers

24 recognizing TTTV and TTV PAM sequences, respectively, in the Mrvi1 locus (Table 1,

25 Supplementary Fig. 1C, F). Out of 5 founders, one was edited with the spacer

26 recognizing the TTTV PAM sequence (20\%), but none from the TTV PAM sequence

27 (Table 1, Supplementary Fig. 1C, F), suggesting that Mb3Cas12a prefers the spacer targeting the TTTV PAM sequence.

As the length of crRNAs has been shown to affect the efficiency of Cas12a-

30 mediated genome editing (Tu et al., 2017; Zetsche et al., 2015), we further tested

31 whether the editing efficiency can be improved by optimizing the crRNA structure. We 
1 first tested effects of the length of crRNAs on genome editing in Dnmt1 locus in HEK293

2 cells (Fig. 1A, B). No Mb3Cas12a activity was detected when a 17nt spacer was used,

3 whereas the highest Mb3Cas12a activities comparable to AsCas12a and LbCas12a

4 were observed in the crRNAs with 23nt spacer (Fig. 1B). Similar results have been

5 reported for AsCas12a and LbCas12a, but not for FnCas12a, which appears to use

6 21nt crRNAs more efficiently (Tu et al., 2017). To determine the potential effects of

7 microinjection (cytoplasmic vs. pronuclear) methods on targeting efficiency, we injected

$8 \mathrm{Mb} 3 \mathrm{Cas} 12 \mathrm{a}$ mRNA and one crRNA, harboring two spacers (one recognizing TTTV

9 PAM sequence, and one TTV) targeting Kcnj10 locus, into either cytoplasm only or both

10 pronucleus and cytoplasm. Interestingly, the cytoplasmic injection appeared to have a

11 higher targeting efficiency $(77 \%, n=20)$ than the pronuclear and cytoplasmic injection

$12(28.6 \%, n=39)$ (Table 1, Fig. 1C, D). Therefore, we used 23nt spacer and cytoplasmic

13 microinjection to generate the following indels in mice (Fig. 2A).

14 To explore whether Mb3Cas12a could target multiple loci simultaneously, we used one crRNA harboring three 23nt spacers recognizing TTTV PAM sequencing targeting the Prps family, i.e. Prps1, Prps2 and Prps1/1. Eleven founders obtained

17 (100\%) were edited by both the Prps1 and Prps1/1 crRNAs, whereas one (9.1\%) was

18 edited only by the Prps2 crRNA (Table 1, Supplementary Fig. 2A), which may reflect a lower targeting efficiency of this particular crRNA. Next, we used a crRNA containing

20 three 23nt spacers separated by 20nt direct repeats to target miR-10b (Table 1,

21 Supplementary Fig. 2B, C). Two of the spacers (spacers 2 and 3) were designed to

22 recognize a TTTV PAM sequence, whereas the other one targets a TTV PAM sequence

23 (spacer 1) at miR-10b locus. All the five founders were edited by the two spacers

24 targeting the TTTV PAM sequence (100\%), and one of them was edited by the one

25 recognizing the TTV PAM sequence (20\%) (Table 1, Supplementary Fig. 2B, C). Similar

26 results were also obtained when we used one crRNA containing one 23nt spacer

27 targeting miR-547 with a TTTV PAM sequence and two 23nt spacers targeting miR-509 with one TTTV PAM and one TTV PAM. All six founders were edited by the two spacers targeting the TTTV PAM sequence (100\%), and one of them was edited by the one recognizing the TTV PAM sequence (16.7\%) (Table 1, Fig. 2B, C). Moreover, although the 23nt spacers recognizing TTTV PAM sequence often led to bi- or multi- 
1 allelic targeting and TTV PAM sequence tended to yield mono-allelic targeting, the 20nt

2 spacers appeared to cause mostly mono-allelic mutations (Table 1, Fig. 1D, 2C and

3 Supplementary Fig. 1, 2). Recent reports have shown that Cas9 with one single gRNA

4 tends to induce large indels in genomic DNA in mouse embryonic stem (ES) cells,

5 progenitor cells and zygotes (Adikusuma et al., 2018; Kosicki et al., 2018), whereas

6 Cas9 with two gRNAs causes large deletions within the two flanking gRNA-targeting

7 sites (Wang et al., 2018). The incidences of large deletions induced by Cas 9 with one

8 single gRNA were $35.7 \%, 36.5 \%$, and $45 \%$ in mouse ES cells, progenitor cells, and

9 zygotes, respectively, whereas the incidence of large insertions was $26.3 \%$ in mouse

10 ES cells (Adikusuma et al., 2018; Kosicki et al., 2018). Interestingly, unlike Cas9,

11 Mb3Cpf-based genome editing predominantly generates indels, containing

12 microhomology $(\mathrm{MH})$ sequences flanking the cleavage sites with one or more spacers

13 within a single crRNA. Among all 42 pups derived from Mb3Cas12a-based editing, only

143 contained large insertions (7.1\%) induced by 3 spacers in the crRNA, whereas the

15 rest displayed different alleles with either two or more small mutations or one mutant

16 plus one wild-type alleles (Table 1, Fig. 1D, 2C and Supplementary Fig. 1, 2). Based on

17 RepeatMasker, two large insertions correspond to ERVL (endogenous retroviruses

18 type-L) and ERVL-MaLR (mammalian apparent LTR retrotransposon), respectively,

19 similar phenomenon has been observed in SpCas9 induced double strand breaks

20 (DSBs) (Ono et al., 2015), suggesting these retrotransposons may hijack the DSBs

21 induced by SpCas9 or Mb3Cas12a. None of the Mb3Cas12a-edited alleles contained

22 large deletions, whereas they are commonly seen in Cas9-editted genes. These results

23 suggest that MMEJ repair mechanism is preferentially adopted in fixing the staggered

24 DNA ends, which may account for the minimal on-target effects in Mb3Cas12a-based

25 genome editing.

Given that two-cell homologous recombination (2C-HR)-CRISPR, in which Cas9

27 was tethered with monomeric streptavidin (mSA) that could bind to biotinylated DNA

28 donor template, showed a higher knock-in (KI) efficiency (Gu et al., 2018), we tested

29 whether Mb3Cas12a-mSA could do the same in generating $\mathrm{KI}$ mice (Fig. 3A, B). We

30 microinjected 2-cell embryos with Mb3Cas12a-mSA mRNA, crRNA targeting Slit2 locus,

31 and biotinylated DNA donor template containing a BamHI restriction enzyme cutting site 
1 (Fig. 3A). Among 5 founders, 2 (40\%) have the knock-in alleles, indicating the

2 Mb3Cas12a-mSA indeed can generate KI mice efficiently (Fig. 3B, 3C). During

3 preparation of our manuscript, one study reporting that HkCas12a can target YTV and

4 TYYN PAM sequences in human cell lines was published (Teng et al., 2019). However,

5 it remains unknown whether HkCas12a works in murine zygotes and what its efficiency

6 is. In summary, our data demonstrate that Mb3Cas12a can edit the murine genome

7 independent of TTTV PAM sequence and with minimal on-target mutations and very

8 high targeting efficiency. Mb3Cas12a-mediated genome editing expands the toolkit for

9 efficient production of mutant mouse lines.

\section{Materials and Methods}

\section{Plasmids construction}

16 To prepare pcDNA3.1-Mb3Cas12a-mSA plasmid, monomeric streptavidin (mSA) DNA

17 fragments amplified from PCS2+Cas9-mSA plasmid (Cat. 103882, Addgene,

18 Watertown, MA) were inserted into the pY117 plasmid (pcDNA3.1-huMb3Cpf1) (Cat. 92293, Addgene) via BamHI (Cat. R0136S, NEB, Ipswich, MA) and EcoRI (Cat. R3101S, NEB) restriction sites.

For the pUC-Slit2-BamHI plasmid, two homology arms ( 1kb) flanking the

22 crRNAs cutting sites of Slit2 locus and pUC empty vector were amplified by Q5® Hot

23 Start High-Fidelity 2X Master Mix (Cat. M0494S, NEB) from mouse tail genomic DNA

24 and pX330 plasmid (Cat. 42230, Addgene), respectively. After purification with Ampure

25 beads, these three DNA fragments were assembled with NEBuilder® HiFi DNA

26 Assembly Master Mix (Cat. E2621L, NEB). BamHI restriction site was introduced

27 between the two homology arms during the PCR amplification. The primers used for

28 plasmids construction are listed in Supplemental Table S1. 
1 To synthesize Mb3Cas12a and Mb3Cas12a-mSA mRNAs, the pY117 plasmid

2 (pcDNA3.1-huMb3Cpf1) (Cat. 92293, Addgene) and pcDNA3.1-Mb3Cas12a-mSA were

3 digested with EcoR I (Cat. R3101S, NEB) overnight at $37^{\circ} \mathrm{C}$, followed by purification

4 with Ampure beads and mRNA synthesis with the HiScribe ${ }^{\mathrm{TM}}$ T7 ARCA mRNA Kit (Cat.

5 E2065S, NEB). Then the in vitro transcribed mRNAs were treated with DNase I (NEB,

6 Cat. M0303S) to remove the plasmid DNA template, followed by poly(A) tailing using $\mathrm{E}$.

7 coli poly(A) polymerase (Cat. M0276S, NEB). The poly(A)-tailed Mb3Cas12a mRNAs

8 were purified using the RNA Clean \& Concentrator ${ }^{\mathrm{TM}}-5$ (Cat. R1016, Zymo Research,

9 Irvine, CA) and eluted in a Tris-EDTA solution (Cat.11-01-02-02, IDT, Coralville, IA).

crRNAs were designed using Benchling (https://benchling.com/). DNA oligos for

11 making each crRNA were synthesized in the IDT Inc. and listed in Supplemental Table

12 S1. To prepare crRNAs for microinjection, the T7 first strand primer and antisense

13 oligos specific for each crRNA were mixed in 1X T4 DNA ligase buffer and heated to

$1495^{\circ} \mathrm{C}$ for 5 minutes, and then allowed to cool down to room temperature on the bench.

15 The annealed oligos were used as the templates for in vitro transcription (IVT) using the

16 HiScribe $^{\text {TM }}$ T7 High Yield RNA Synthesis Kit (Cat. E2040S, NEB). After IVT, crRNAs

17 were purified using the RNA Clean \& Concentrator ${ }^{\mathrm{TM}}-5$ (Cat. R1016, Zymo Research)

18 and eluted in Tris-EDTA solution (Cat.11-01-02-02, IDT).

To prepare crRNAs for transfection of HEK293 cells, PCR products

20 corresponding to each crRNA were amplified with U6 forward primer and corresponding

21 antisense oligos (as listed in Supplemental Table S1) from the pX330 plasmid (Cat.

22 42230, Addgene). After digestion with Dpnl (Cat. R0176S, NEB), the PCR products

23 were purified using Ampure beads.

24 The biotinylated donor DNA template was amplified from the pUC-Slit2-BamHI

25 plasmid with biotinylated primers (as listed in Supplemental Table S1), followed by

26 Dpnl digestion to remove the plasmid and purification with Ampure beads.

HEK293 cells Transfection

29 HEK293 cells were co-transfected with 400ng of pY117 (pcDNA3.1-huMb3Cpf1) (Cat.

30 92293, Addgene) and 100ng of crRNA PCR product using Lipofectamine 2000 (Cat. 
1 11668, Thermo Fisher Scientific, Waltham, MA) in a 24 well cell culture plate (Cat.

23524 , Corning, Corning, NY). After 48h, cells were collected for analyses.

4 Animal use and generation of knockout (KO) and knock-in (KI) mice

5 The animal protocol for this study was approved by the Institutional Animal Care and

6 Use Committee (IACUC) of the University of Nevada, Reno (protocol number 00494).

7 All mice were housed and maintained under specific pathogen free conditions with a

8 temperature- and humidity-controlled animal facility in the Department of Lab Animal

9 Medicine, University of Nevada, Reno. Generation of KO and KI mice were performed

10 as previously described with modifications (Gu et al., 2018; Wang et al., 2018; Wang et

11 al., 2019). Briefly, 4-6 weeks of FVB/NJ or C57BL/6J female mice were super-ovulated

12 and mated with C57BL/6J stud males; zygotes and 2-cell stage embryos were collected

13 from the oviducts for $\mathrm{KO}$ and $\mathrm{KI}$, respectively. For KO, Mb3Cas12a mRNA (200ng/ $\mu \mathrm{l})$

14 and crRNA $(100 \mathrm{ng} / \mu \mathrm{l})$ were mixed and injected into the cytoplasm or pronucleus of the

15 zygotes in M2 medium (Cat. MR-051-F, Millipore, Burlington, MA). For KI, Mb3Cas12a-

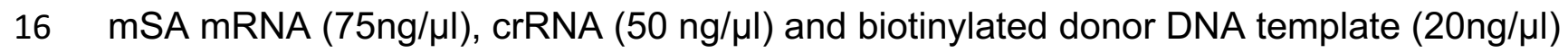

17 were mixed and injected into the cytoplasm or pronucleus of the 2-cell embryos in M2

18 medium. After injection, all embryos were cultured for $1 \mathrm{~h}$ in KSOM+AA medium (Cat. MR-121-D, Millipore) at $37^{\circ} \mathrm{C}$ under $5 \% \mathrm{CO}_{2}$ in air before being transferred into $7-10$

20 week-old female CD1 recipients.

\section{Mouse genotyping, T7El and Sanger sequencing}

23 Mouse genotyping was performed as previously described (Wang et al., 2018; Wang et

24 al., 2019). Briefly, mouse tail or ear snips were lysed in a lysis buffer $(40 \mathrm{mM} \mathrm{NaOH}$,

$250.2 \mathrm{mM}$ EDTA) for $1 \mathrm{~h}$ at $95^{\circ} \mathrm{C}$, followed by neutralization using the same volume of

26 neutralizing buffer $(40 \mathrm{mM}$ Tris- $\mathrm{HCl})$. PCR reactions were conducted using Platinum ${ }^{\mathrm{TM}}$

27 SuperFi ${ }^{\mathrm{TM}}$ Green PCR Master Mix (Cat. 12359010, Thermo Fisher Scientific). T7EI

28 (Cat. M0302L, NEB) assay was followed to detect the mutations. The positive samples were proceeded with A tailing using GoTaq® Green Master Mix (Cat. M7123, Promega,

30 Madison, $\mathrm{WI}$ ) for $5 \mathrm{~min}$ at $95^{\circ} \mathrm{C}$, followed by $15 \mathrm{~min}$ at $72{ }^{\circ} \mathrm{C}$. The A-tailed PCR products 31 were then ligated to $\mathrm{pGEM} 囚-T$ Easy Vector using pGEM®-T Easy Vector Systems (Cat. 
1 A1360, Promega). Positive colonies were selected for Sanger sequencing. Data was

2 analyzed using Geneious software. The primers used for genotyping are listed in

3 Supplemental Table S1.

4

5 MiSeq library construction and analysis

6 DNA fragment of Prps1, Prps2, and Prps1/1 were amplified using Platinum ${ }^{\mathrm{TM}}$ SuperFi ${ }^{\mathrm{TM}}$

7 Green PCR Master Mix (Cat. 12359010, Thermo Fisher Scientific) from lysis of mouse

8 tail or ear snips. The PCR products were then tagged using Nextera XT DNA Library

9 Preparation Kit (Cat. 15032354, Illumina, San Diego, CA) and indexed using Nextera

10 XT Index Kit (Cat. 15055294, Illumina). DNA library was sequenced using MiSeq

11 Reagent Kit v2 (500-cycles) (Cat. MS-102-2003, Illumina). Data was analyzed using

12 Geneious software. The primers used for Prps1, Prps2, and Prps1/1 are listed in

13 Supplemental Table S1.

15 Acknowledgements

16 Not applicable.

18 Competing interests

19 No competing interests declared.

21 Funding

22 This work was supported by grants from the National Institutes of Health (HD071736,

23 HD085506, and P30GM110767 to WY) and John Templeton Foundation (PID: 61174 to 24 WY).

\section{Data availability}

27 The datasets generated and/or analyzed during the current study are available in the

28 Sequence Read Archive (SRA), https://www.ncbi.nlm.nih.gov/sra/PRJNA556550 29 
bioRxiv preprint doi: https://doi.org/10.1101/731646; this version posted August 10,2019 . The copyright holder for this preprint (which was not certified by peer review) is the author/funder, who has granted bioRxiv a license to display the preprint in perpetuity. It is made available under aCC-BY-NC-ND 4.0 International license.

1 Z. W. and W. Y. conceived and designed the research. Z. W., Y. W., S. W., A. J G., H. 2 M., T. Y., K. C-G., and H.Z. performed bench experiments. Z. W. analyzed data. Z. W.

3 and W. Y. wrote the manuscript. All reviewed and agreed with the contents of the

4 manuscript.

5 
Reference:

2 Adikusuma, F., Piltz, S., Corbett, M. A., Turvey, M., McColl, S. R., Helbig, K. J., Beard, M. R., Hughes, J., Pomerantz, R. T. and Thomas, P. Q. (2018). Large deletions induced by Cas9 cleavage. Nature 560, E8-E9.

Fu, Y., Foden, J. A., Khayter, C., Maeder, M. L., Reyon, D., Joung, J. K. and

Fonfara, I., Richter, H., Bratovic, M., Le Rhun, A. and Charpentier, E. (2016). The CRISPR-associated DNA-cleaving enzyme Cpf1 also processes precursor

Cong, L., Ran, F. A., Cox, D., Lin, S. L., Barretto, R., Habib, N., Hsu, P. D., Wu, X. B., Jiang, W. Y., Marraffini, L. A., et al. (2013). Multiplex Genome Engineering Using CRISPR/Cas Systems. Science 339, 819-823.

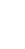

$$
\text { CRISPR RNA. Nature 532, 517-521. }
$$
Sander, J. D. (2013). High-frequency off-target mutagenesis induced by CRISPR-Cas nucleases in human cells. Nat Biotechnol 31, 822-826.

Gu, B., Posfai, E. and Rossant, J. (2018). Efficient generation of targeted large insertions by microinjection into two-cell-stage mouse embryos. Nat Biotechnol 36, 632-637.

Hsu, P. D., Scott, D. A., Weinstein, J. A., Ran, F. A., Konermann, S., Agarwala, V., Li, Y., Fine, E. J., Wu, X., Shalem, O., et al. (2013). DNA targeting specificity of RNA-guided Cas9 nucleases. Nat Biotechnol 31, 827-832.

Jinek, M., Chylinski, K., Fonfara, I., Hauer, M., Doudna, J. A. and Charpentier, E. (2012). A Programmable Dual-RNA-Guided DNA Endonuclease in Adaptive Bacterial Immunity. Science 337, 816-821.

Kim, H. K., Song, M., Lee, J., Menon, A. V., Jung, S., Kang, Y. M., Choi, J. W., Woo, E., Koh, H. C., Nam, J. W., et al. (2017). In vivo high-throughput profiling of CRISPR-Cpf1 activity. Nat Methods 14, 153-159.

Kleinstiver, B. P., Tsai, S. Q., Prew, M. S., Nguyen, N. T., Welch, M. M., Lopez, J. M., McCaw, Z. R., Aryee, M. J. and Joung, J. K. (2016). Genome-wide specificities of CRISPR-Cas Cpf1 nucleases in human cells. Nat Biotechnol 34, 869-874.

Komor, A. C., Badran, A. H. and Liu, D. R. (2017). CRISPR-Based Technologies for the Manipulation of Eukaryotic Genomes. Cell 169, 559. 
Kosicki, M., Tomberg, K. and Bradley, A. (2018). Repair of double-strand breaks induced by CRISPR-Cas9 leads to large deletions and complex rearrangements. Nat Biotechnol 36, 765-771.

Lee, H. and Kim, J. S. (2018). Unexpected CRISPR on-target effects. Nat Biotechnol 36, 703-704.

Ono, R., Ishii, M., Fujihara, Y., Kitazawa, M., Usami, T., Kaneko-Ishino, T., Kanno, J., Ikawa, M. and Ishino, F. (2015). Double strand break repair by capture of retrotransposon sequences and reverse-transcribed spliced mRNA sequences in mouse zygotes. Scientific reports 5, 12281.

Strohkendl, I., Saifuddin, F. A., Rybarski, J. R., Finkelstein, I. J. and Russell, R. (2018). Kinetic Basis for DNA Target Specificity of CRISPR-Cas12a. Mol Cell.

Teng, F., Li, J., Cui, T., Xu, K., Guo, L., Gao, Q., Feng, G., Chen, C., Han, D., Zhou, Q., et al. (2019). Enhanced mammalian genome editing by new Cas12a orthologs with optimized crRNA scaffolds. Genome Biol 20, 15.

Tu, M., Lin, L., Cheng, Y., He, X., Sun, H., Xie, H., Fu, J., Liu, C., Li, J., Chen, D., et al. (2017). A 'new lease of life': FnCpf1 possesses DNA cleavage activity for genome editing in human cells. Nucleic acids research 45, 11295-11304.

Wang, H., Yang, H., Shivalila, C. S., Dawlaty, M. M., Cheng, A. W., Zhang, F. and Jaenisch, R. (2013). One-step generation of mice carrying mutations in multiple genes by CRISPR/Cas-mediated genome engineering. Cell 153, 910-918.

Wang, Z., Lee, S., Oliver, D., Yuan, S., Tang, C., Wang, Y., Zheng, H. and Yan, W. (2018). Prps1l1, a testis-specific gene, is dispensable for mouse spermatogenesis. Mol Reprod Dev 85, 802-804.

Wang, Z., McSwiggin, H., Newkirk, S. J., Wang, Y., Oliver, D., Tang, C., Lee, S., Wang, S., Yuan, S., Zheng, H., et al. (2019). Insertion of a chimeric retrotransposon sequence in mouse Axin1 locus causes metastable kinky tail phenotype. Mobile DNA 10, 17.

Zetsche, B., Gootenberg, J. S., Abudayyeh, O. O., Slaymaker, I. M., Makarova, K. S., Essletzbichler, P., Volz, S. E., Joung, J., van der Oost, J., Regev, A., et al. (2015). Cpf1 Is a Single RNA-Guided Endonuclease of a Class 2 CRISPRCas System. Cell 163, 759-771. 
1 Zetsche, B., Heidenreich, M., Mohanraju, P., Fedorova, I., Kneppers, J.,

2 DeGennaro, E. M., Winblad, N., Choudhury, S. R., Abudayyeh, O. O.,

Gootenberg, J. S., et al. (2017a). Multiplex gene editing by CRISPR-Cpf1 using a single crRNA array. Nat Biotechnol 35, 31-34.

5 Zetsche, B., Strecker, J., Abudayyeh, O. O., Gootenberg, J. S., Scott, D. A. and Zhang, F. (2017b). A Survey of Genome Editing Activity for 16 Cpf1 orthologs. bioRxiv 
1 Figure 1. Optimization of Mb3Cas12a-based genome editing efficiency by

2 adjusting the crRNA length and microinjection sites.

3 A. Sequences of crRNAs targeting Dnmt1 locus. crRNAs with one 20nt direct repeat

4 and spacers of various lengths recognizing TTTV PAM sequences were used for

5 targeting Dnmt1 in HEK293 cells. B. T7El assay results of Mb3Cas12a-edited Dnmt1

6 locus in HEK293 cells. C. PCR and PCR-T7EI to identify the efficiency of Mb3Cas12a-

7 based genome editing in Kcnj10 locus by either pronuclear and cytoplasmic

8 microinjection or cytoplasmic microinjection only in murine zygotes. D. crRNAs used in

9 targeting Kcnj10 locus (upper panel) and Sanger sequencing results of Mb3Cas12a-

10 based genome editing in Kcnj10 locus in murine zygotes (lower panel). Red underlines

11 represent microhomology $(\mathrm{MH})$ sequences.

Figure 2. Multiplex targeting efficiency of Mb3Cas12a in miR-547 \& miR-509 loci

14 with TTV PAM sequence in mice.

15 A. Schematics showing the strategy used for generating indels in mouse zygotes.

$16 \mathrm{Mb3Cas12a}$ mRNA and 23nt crRNA are microinjected into the cytoplasm of mouse

17 zygotes. B. PCR genotyping results of Mb3Cas12a-edited miR-547 \& miR-509

18 founders. One crRNA with one 23nt spacer targeting miR-547 with a TTTV PAM sequence and two 23nt spacers targeting miR-509 with one TTTV PAM and one TTV

20 PAM sequences was used to target miR-547 \& miR-509 loci. C. Sanger sequencing

21 results of Mb3Cas12a-edited pups \#1, \#2, \#3, \#4, \#5 and \#6 in miR-547 \& miR-509 loci.

22 Red underlines represent microhomology $(\mathrm{MH})$ sequences, characters in grey

23 background indicate large insertions.

Figure. 3 Generation of knock-in (KI) in Slit2 locus using Mb3Cas12a-mSA in 26 mouse 2-cell embryos.

27 A. Schematics showing the strategy used for generating $\mathrm{KI}$ in 2-cell mouse embryos. Mb3Cas12a mRNA, crRNA and biotinylated donor DNA template are microinjected into 2-cell mouse embryos. B. Efficiency of Mb3Cas12a-mSA-mediated KI in Slit2 locus in

30 mice. One crRNA harboring two 23nt spacers recognizing one TTTV PAM and one

31 TTV PAM sequence was used to target Slit2 locus. Upper panel, strategy used for 
1 generating Slit2-BamHI KI. Colored characters represent DNA sequence, black

2 characters in colored background indicate corresponding amino acids. Lower panel,

3 PCR, PCR-T7EI (T7 endonuclease I assay) and PCR-BamHI digestion show the KI

4 efficiency. C. Sanger sequencing results of Mb3Cas12a-mSA-mediated KI pups \#2, and

$5 \quad \# 4$ in Slit2 locus.

Supplementary Figure 1. Efficiency of Mb3Cas12a-based genome editing using

8 20nt spacers targeting Prps1/1, Saraf and Mrvi1 loci in mice.

9 A. T7 endonuclease I (T7EI) assay results of Mb3Cas12a-edited Prps1/1 locus. One

10 crRNA with one 20nt direct repeat and one 20nt spacer recognizing a TTTV PAM

11 sequence was used for targeting Prps1/1. B. T7El assay results of Mb3Cas12a-edited

12 Saraf locus. Two crRNAs harboring two 20nt spacers either recognizing two TTTV or

13 two TTV PAM sequences were used to target Saraf locus. The expected bands

14 corresponding to the pups after T7EI assays are indicated with the same color. C. T7EI

15 assay results of Mb3Cas12a-edited Mrvi1 locus. One single crRNA containing two

16 spacers recognizing both TTTV and TTV PAM sequences was used to target Mrvi1

17 locus. D. Sanger sequencing results of Mb3Cas12a-edited pup \#23 in Prps1/1 locus. E.

18 Sanger sequencing results of Mb3Cas12a-edited pups \#15 and \#21 in Saraf locus. F.

19 Sanger sequencing results of Mb3Cas12a-edited pup \#16 in Mrvi1 locus. Red

20 underlines represent microhomology $(\mathrm{MH})$ sequences.

\section{Supplementary Figure 2. Multiplex targeting efficiency of Mb3Cas12a in Prps}

23 family and miR-10b loci in mice. A. MiSeq results of Mb3Cas12a targeting efficiency

24 in Prps1, Prps2, and Prps1/1 with one crRNA, which contains three 20nt direct repeats

25 and three 23nt spacers targeting Prps1, Prps2 and Prps1/1 with TTTV PAM sequences.

26 Red underlines represent microhomology $(\mathrm{MH})$ sequences. B. PCR genotyping results

27 of Mb3Cas12a-edited miR-10b founders. One crRNA with three 20nt direct repeats and

28 three 23nt spacers recognizing two TTTV PAM and one TTV PAM sequences was used

29 to target miR-10b locus. C. Sanger sequencing results of Mb3Cas12a-edited pups \#1,

30 \#2, \#3, \#4 and \#5 in miR-10b locus. 
bioRxiv preprint doi: https://doi.org/10.1101/731646; this version posted August 10, 2019. The copyright holder for this preprint (which was not certified by peer review) is the author/funder, who has granted bioRxiv a license to display the preprint in perpetuity. It is made available under

\section{Figure 1} aCC-BY-NC-ND 4.0 International license.

A

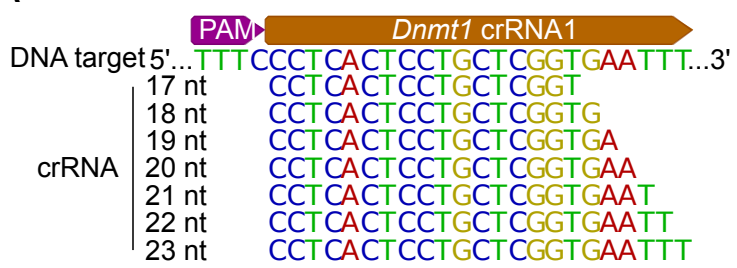

B

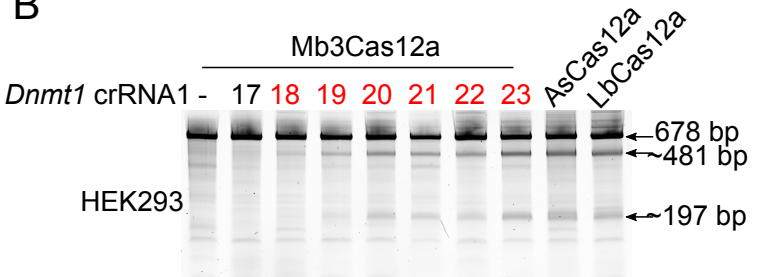

Cytoplasmic Microinjection

C

Pronuclear \& Cytoplasmic Microinjections 3 $2627282930313233 \quad 343536 \quad \mathrm{H}_{2} \mathrm{O}$ Animal \# $10 \quad 1112 \quad 13 \quad 141516 \quad 171819202122 \quad 3$ 42526

PCR

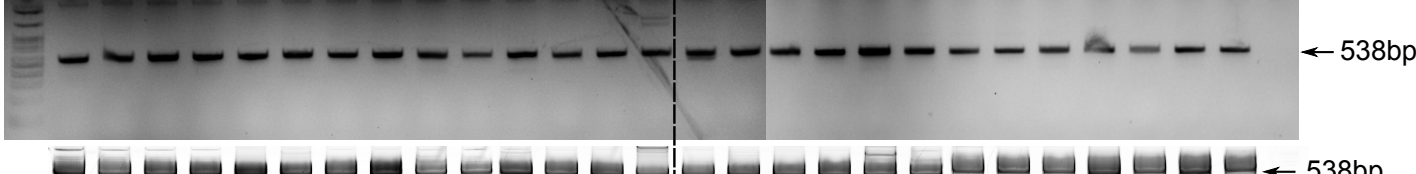

T7EI

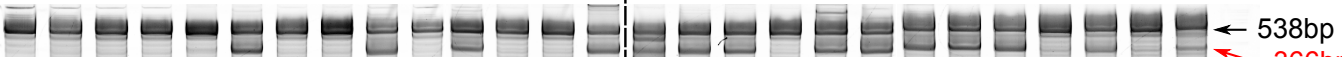

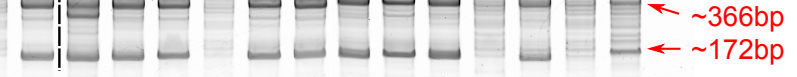

D

\section{Kcnj10 gene}

Kcnj10 CDS

5'... AATAACTTTCTATATGCCATTTTACTTCCTGCAGATGACGTCGGTCGCTAAGGTCTATTA ...3'

3'... TTATTGAAAGATATACGGTAAAATGAAGGACGTCTACTGCAGCCAGCGATTCCAGATAAT ...5' Kcnj10 TTcrRNA3 PAM

p15 AATAACTTTCTATATGCCATTTTACT--- - -GCAGATGACGTCGGTCGCTAAGGTCTATTA -4

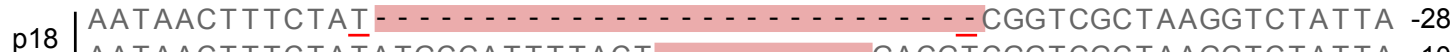

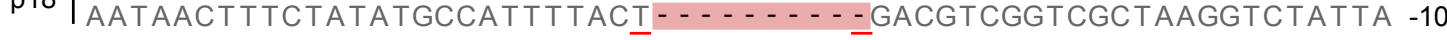

p20 AATAACTTTCTATATGCCATTTTAC- - -CTGCAGATGACGTCGgTCGCTAAgGTCTATTA -3

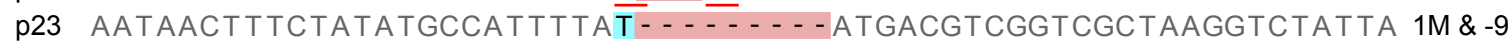

p24 AATAACTTTCTATATGCCATTTTAC-...-...-. ATGACGTCGGTCGCTAAGGTCTATTA

p25 AATAACTTTCTATATGCCATTTTACTTCㄷ- - - AGATGACGTCGGTCGCTAAGGTCTATTA -3

p26 AATAACTTTCTATATGCCATTTTACT- - - - GCAGATGACGTCGgTCGCTAAGgTCTATTA -4

p28 AATAACTTTCTATATGCCATTTTAT- - - - - A ATGACGTCGGTCGCTAAGGTCTATTA $1 \mathrm{M} \&-9$

p29 AATAACTTTCTATATGCCATTTTATTTCC-.-- A ATGACGTCGGTCGCTAAGGTCTATTA 1 M \& -5

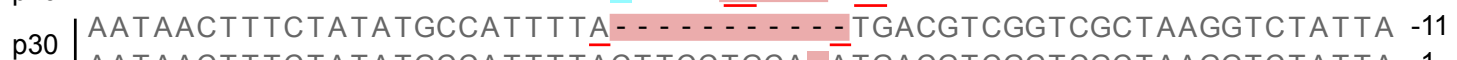

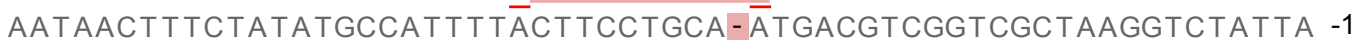

p31 AATAACTTTCTATATGCCATTTTACTTCCGG--GATGACGTCGGTCGCTAAGGTCTATTA 1M \& -2

p32 AATAACTTTCTATATGCCATTTTACT- - - - GCAGATGACGTCGGTCGCTAAGGTCTATTA

p34 AATAACTTTCTATATGCCATTTTACTTCCTG- - -ATGACGTCGGTCGCTAAGGTCTATTA -3

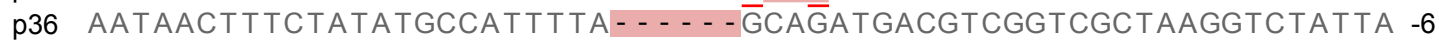


bioRxiv preprint doi: https://doi.org/10.1101/731646; this version posted August 10, 2019. The copyright holder for this preprint (which was not certifiqdiey(perer peview) is the author/funder, who has granted bioRxiv a license to display the preprint in perpetuity. It is made available under aCC-BY-NC-ND 4.0 International license.

A

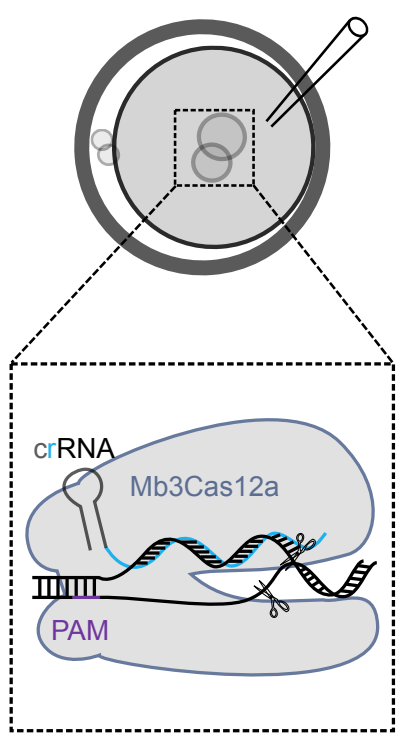

B
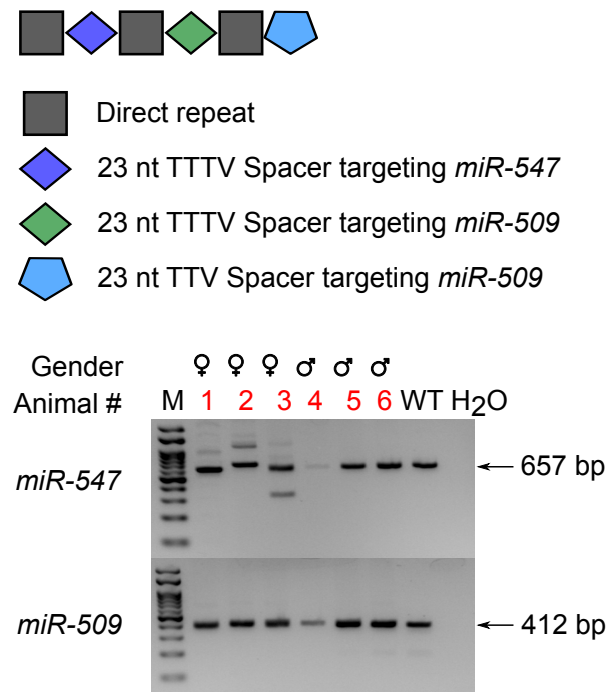

C

5'... GTGTGTTATCTCACTCAAAGATGTACCAAGCATGITTCCTGTTAAATGGGTGGTACATCCTCAAGTGATACATCACAC ....3' miR547 precursor RNA
mmu-miR-547-3p
miR547 crRNA2

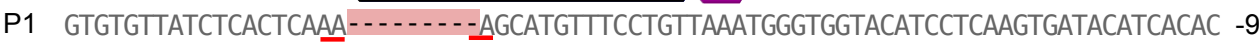

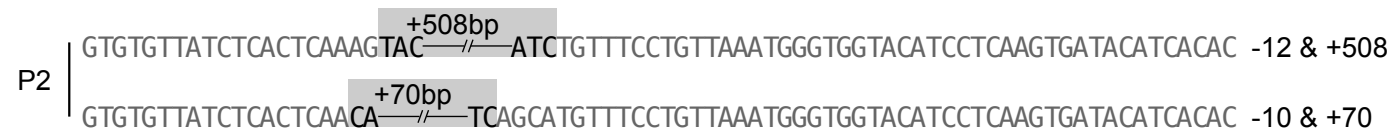

P3 GTGTGTTATCTCACTCAAT- - - TACCAAGCATGTITCCTGTTAAATGGGTGGTACATCCTCAAGTGATACATCACAC $1 \mathrm{~m} \&-4$

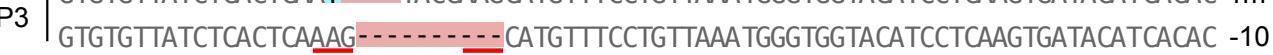

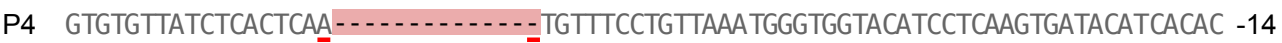

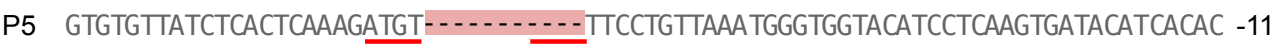

P6 GTGTGTTATCTCACTCAAAGA-...-- GCATGTTCCTGTTAAATGGGTGGTACATCCTCAAGTGATACATCACAC -8

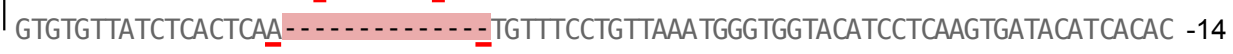

5'... ATGTGTTACTCCATTACAGAAATGTCAATCACATTTAATTATGCATGATTGCCACATTCTGGAGTAAAGAACCAC ...3'

miR509 precursor RNA
mmu-miR-509-3p
miR509_TTCrRNA2

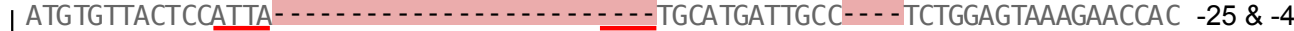

P1 ATGTGTTACTCCATTACAGAAATGTCAATCACATTTAATTATGCATGATTGCCAC- - - - GGAGTAAAGAACCAC -5

ATGTGTTACTCCATTACAGAAATGTCAATCACATTTAATTATGCATGATTG- - - - - - - - GAGTAAAGAACCAC

ATGTGTTACTCCATTACAGAAATG TCAATCACATTTAATTATGCATGATTGCCAC- - - - GGAGTAAAGAACCAC -5

P2 ATGTGTTACTCCATTACAGAAATGTCAATCACATTTAATTATGCATGATTG- - - - - - - -GAGTAAAGAACCAC -10 ATGTGTTACTCCATTACAGAAATGTCAATCACATTTAATTATGCATGATTGC-ACATTCTGGAGTAAAGAACCAC -1

P3 ATG TGTTACTCCATTACAGAAATGTCAATCACATTTAATTATGCATG - - - - - - - -

3 ATGTGTTACTCCATTACAGAAATGTCAATCACATTTAATTATGCATGATTG- - - - -TCTGGAGTAAAGAACCAC -6

P4 ATGTGTTACTCCATTACAGAAATGTCAATCACATTTAATTATGCATGATTGCCA- - TTCTGGAGTAAAGAACCAC -2

P5 ATGTGTTACTCCATTACAGAAATGTCAATCACATTTAATTATGCATGATTGCCAC- - - - GGAGTAAAGAACCAC -5

P6 ATGTGTTACTCCATTACAGAAATGTCAATCACATTTAATTATGCATGATTGCCAC- - - -GGAGTAAAGAACCAC 
bioRxiv preprint doi: https://doi.org/10.1101/731646; this version posted August 10, 2019. The copyright holder for this preprint (which was not certifiqdigy (peer zeview) is the author/funder, who has granted bioRxiv a license to display the preprint in perpetuity. It is made available under

A

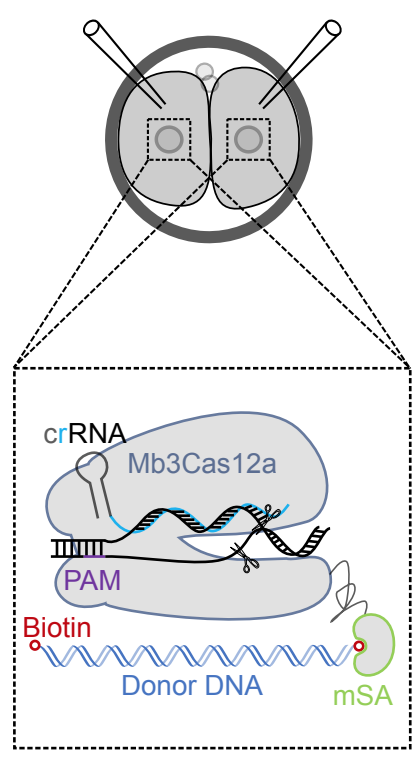

$B$

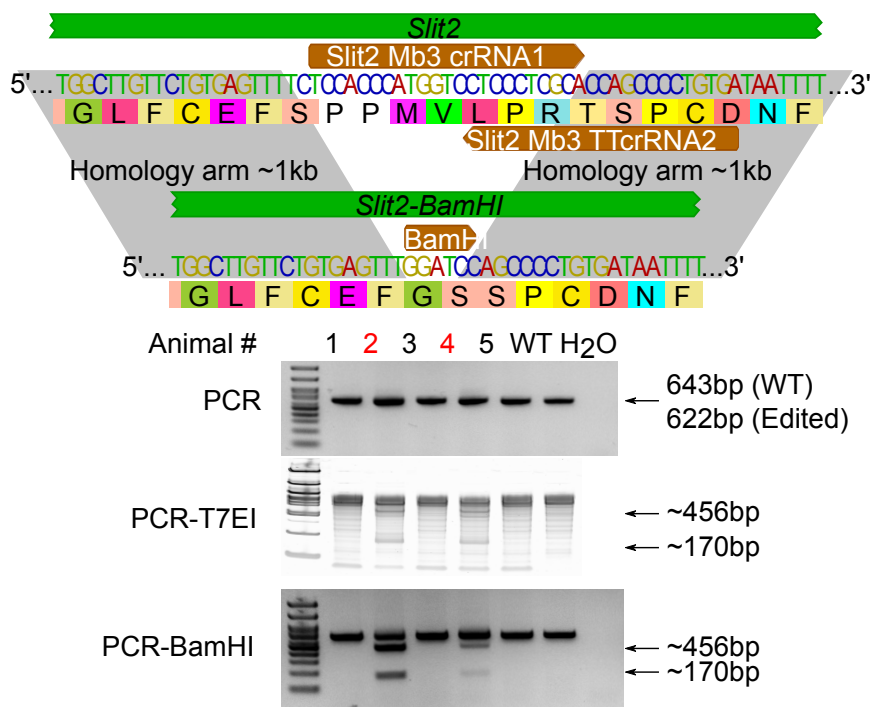

C

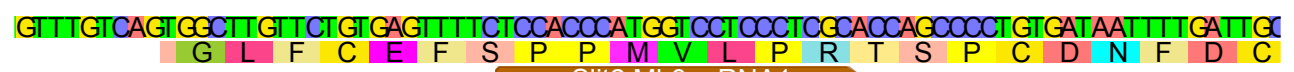
G L F C E F Slit2 Mb3 crRNA1

Slit2 CDS

P2

KI MWMWMWWWMWMM

MWWW WMWMWMWMW

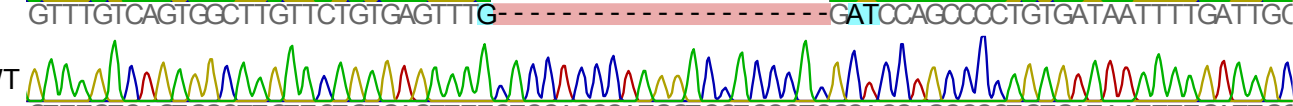
GTTTGTCAGT GGCTT GT TCT GT GAGTTTTCTCCACCCATGGT CCTCCCTCGCACCAGCCCCT GT GATAATTTT GATT GC

P4

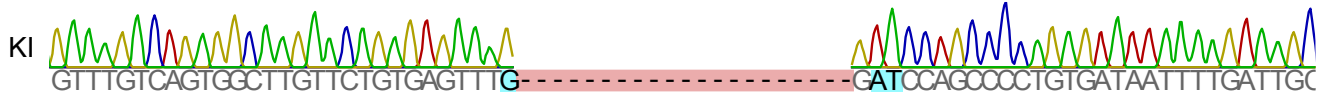

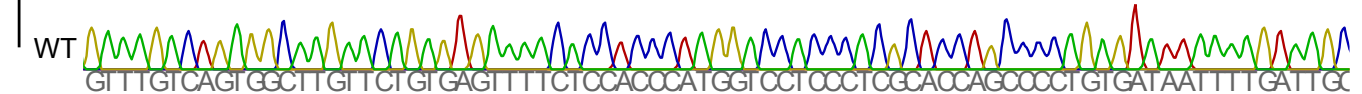


Table 1. Editing efficiency of Mb3Cpf1 in murine zygotes.

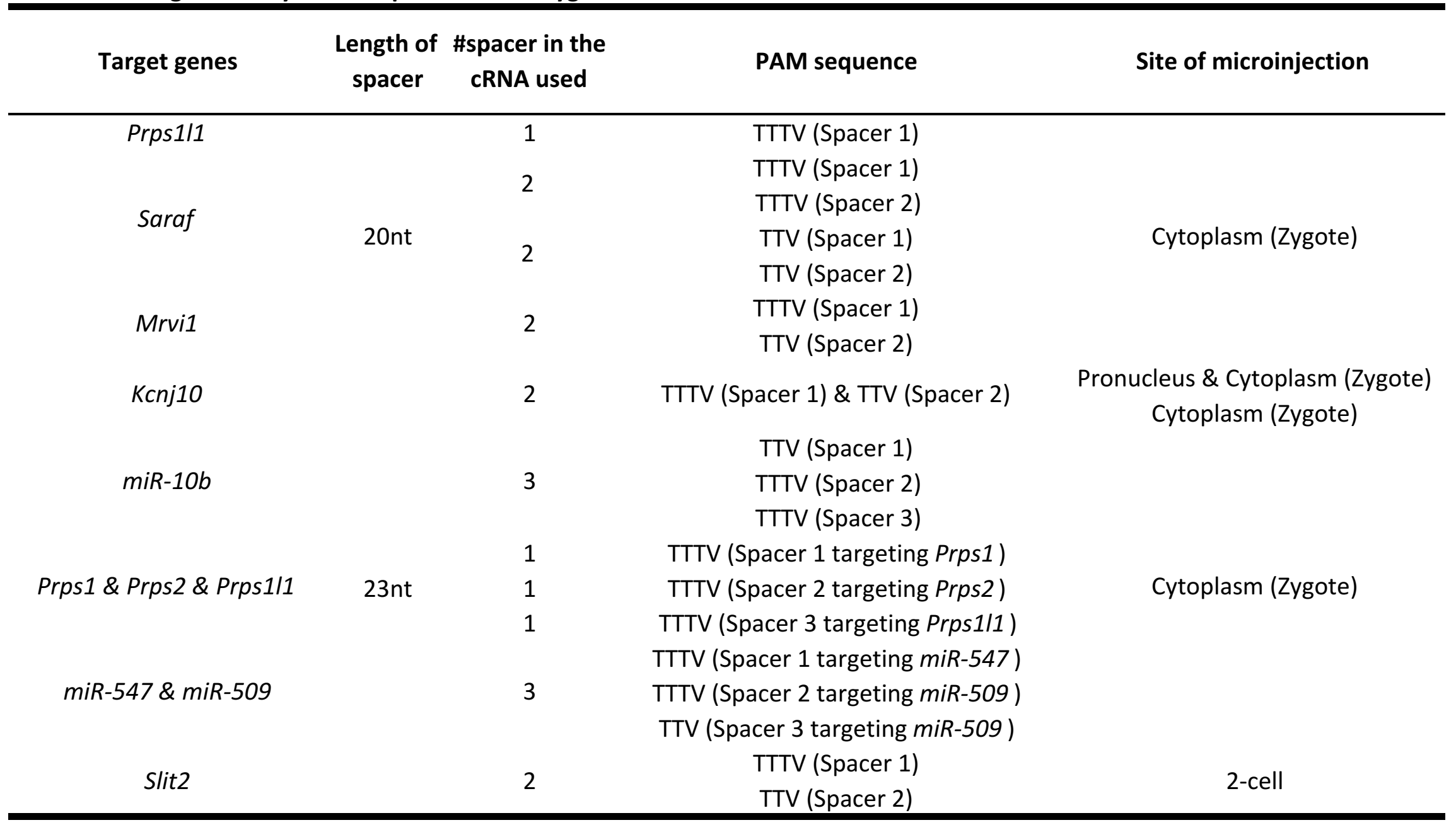

\footnotetext{
${ }^{\mathrm{a}}$ : (\#Newborns/\#Zygotes injected and transferred)*\%

b. (\#Mutants/\#Newborns)*\%
} 


\begin{tabular}{|c|c|c|c|c|c|c|}
\hline $\begin{array}{l}\text { \#Zygotes injected } \\
\text { and transferred }\end{array}$ & $\begin{array}{l}\text { \#Newborns } \\
\text { (birth rate) }^{a}\end{array}$ & $\begin{array}{c}\text { \#Mutants (targeting } \\
\text { efficiency) }^{\mathrm{b}}\end{array}$ & $\begin{array}{l}\text { Indel/Knock- } \\
\text { in (KI) }\end{array}$ & $\begin{array}{c}\text { Mono-allelic } \\
\text { mutation }\end{array}$ & $\begin{array}{l}\text { Bi-allelic } \\
\text { mutation }\end{array}$ & $\begin{array}{c}\text { Multi-allelic } \\
\text { mutation }\end{array}$ \\
\hline 22 & $6(27.3 \%)$ & $1(16.7 \%)$ & \multirow{18}{*}{ Indel } & $Y$ & $\mathrm{~N}$ & $\mathrm{~N}$ \\
\hline \multirow{2}{*}{22} & \multirow{2}{*}{$3(13.6 \%)$} & $1(33.3 \%)$ & & Y & $\mathrm{N}$ & $\mathrm{N}$ \\
\hline & & $0(0 \%)$ & & $N$ & $N$ & $N$ \\
\hline \multirow{2}{*}{21} & \multirow{2}{*}{$4(19 \%)$} & $0(0 \%)$ & & $\mathrm{N}$ & $\mathrm{N}$ & $\mathrm{N}$ \\
\hline & & $1(25 \%)$ & & $Y$ & $\mathrm{~N}$ & $\mathrm{~N}$ \\
\hline \multirow{2}{*}{23} & \multirow{2}{*}{$6(26.1 \%)$} & $1(16.7 \%)$ & & $Y$ & $N$ & $\mathrm{~N}$ \\
\hline & & $0(0 \%)$ & & $N$ & $\mathrm{~N}$ & $\mathrm{~N}$ \\
\hline 39 & 14 (35.9\%) & $4(28.6 \%)$ & & $Y$ & $Y$ & $N$ \\
\hline \multirow[t]{2}{*}{20} & 13 (65\%) & $10(77 \%)$ & & I & r & V \\
\hline & & $1(20 \%)$ & & $Y$ & $\mathrm{~N}$ & $\mathrm{~N}$ \\
\hline \multirow[t]{3}{*}{12} & $5(41.7 \%)$ & $5(100 \%)$ & & $N$ & $Y$ & $\mathrm{~N}$ \\
\hline & & 5 (100\%) & & $N$ & $Y$ & $\mathrm{~N}$ \\
\hline & & $11(100 \%)$ & & $\mathrm{N}$ & $Y$ & $Y$ \\
\hline \multirow[t]{3}{*}{30} & 11 (36.7\%) & $1(9.1 \%)$ & & $N$ & $Y$ & $Y$ \\
\hline & & 11 (100\%) & & $Y$ & $N$ & $N$ \\
\hline & & $6(100 \%)$ & & $N$ & $Y$ & $Y$ \\
\hline \multirow[t]{2}{*}{38} & $6(15.8 \%)$ & $6(100 \%)$ & & $N$ & $Y$ & $Y$ \\
\hline & & 1 (16.7\%) & & $Y$ & $\mathrm{~N}$ & $\mathrm{~N}$ \\
\hline 23 & $5(21.7 \%)$ & $2(40 \%)$ & $\mathrm{KI}$ & & $\mathrm{N} / \mathrm{A}$ & \\
\hline
\end{tabular}


bioRxiv preprint doi: https://doi.org/10.1101/731646; this version posted August 10, 2019. The copyright holder for this preprint (which was not certified by peesquiew) is the author/funder, who has granted bioRxiv a license to display the preprint in perpetuity. It is made available under aCC-BY-NC-ND 4.0 International license.

A

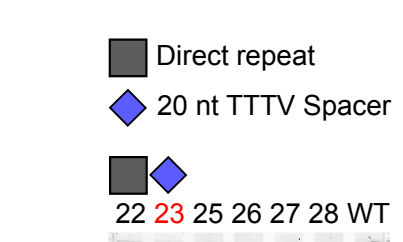

Prps1/1
B

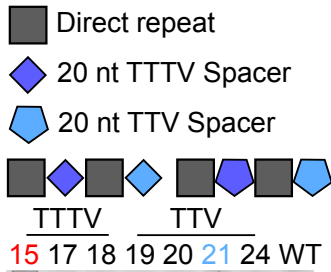

C

Direct repeat

$\checkmark 20 \mathrm{nt}$ TTTV Spacer

$20 \mathrm{nt}$ TTV Spacer

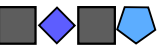

1112131416 WT

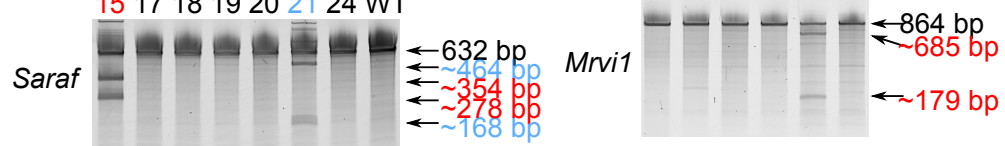

$\mathrm{D}$

5'... 3T TA C T GCAGT CA T C C CGTGCT T C C C T TA T G C C CGGCA GGA TAAAAAGGA 1...3'

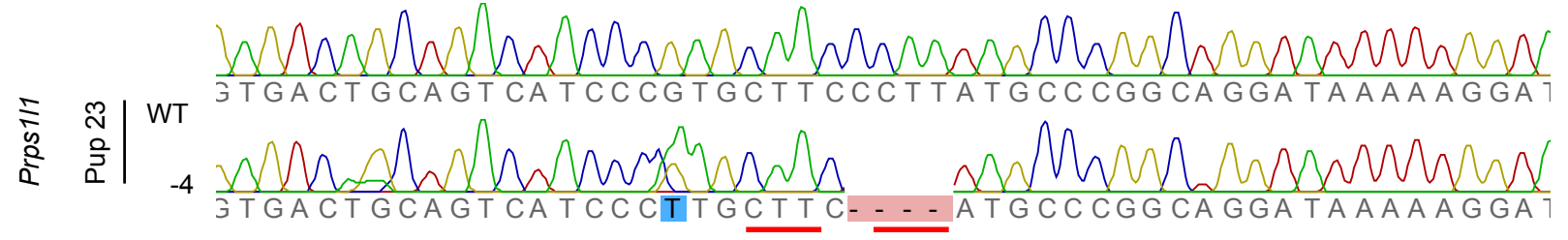

$E$

5'... C T T C T T T CA GA CA GA A T A C T C T T G C G G GA T G T G A A A G C T C T TA C C C T C T A...3' PAM SarafcrinA

:

引 CTTCTTCAGACAGAATACTCTTGCGGGATGTAAAGCTCTTACCCTCTA

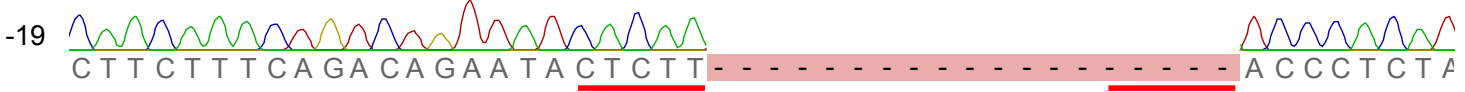

5'... C GC C G G T T G T GA G GC C TA TA C C C C CA G G G T GA T A CA GT GC CA GA A CAA A C...3' Exon2

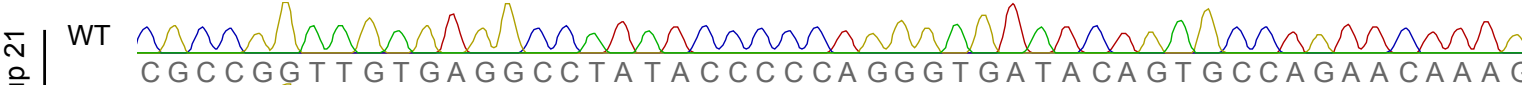 $\stackrel{2}{3}$ -11 mwaman CGCCGGT TGTGAGGCCTA T................. TA CAGTGCCAGAACAAAC

$\mathrm{F}$

5'... AGG C CA CG C C CA T G G C T CA G GCT T C TGGA G CAA GCA T CA T G G GAAAA T G C...3' Mrvi1 crRNA 


\section{Figure S2}

bioRxiv preprint doi: https://doi.org/10.1101/731646; this version posted August 10, 2019. The copyright holder for this preprint (which was not certified by peer review) is the author/funder, who has granted bioRxiv a license to display the preprint in perpetuity. It is made available under A

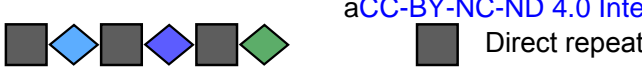

23 nt TTTV Spacer

5'... TTCATTCTCTTTTTTTCTATTTTAGTGTGGAAATTGGCGAAAGCGTTCGTGGAGAAGATGTCTACA ...3' PAM Pros1 crRNA

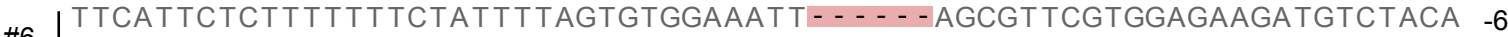

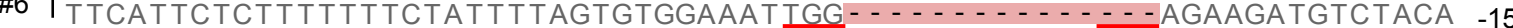

\#7 TTCATTCTCTTTTTTTCTATTTTAGTgTgGAAATGGCG- - - - - TTCGTGGAGAAGATGTCTACA

ITTCATTCTCTTTTTTTCTATTTTAGTGTGGAAATTGGCGAAAG- - TTCGTGGAGAAGATGTCTACA -2

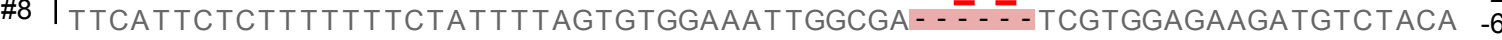

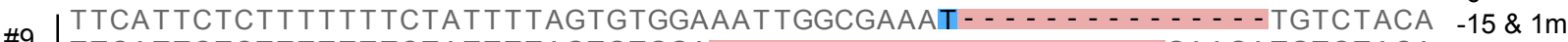

І

\#10 TTCATTCTCTTTTTTTCTATTTTAGTGT"GG'A'AA

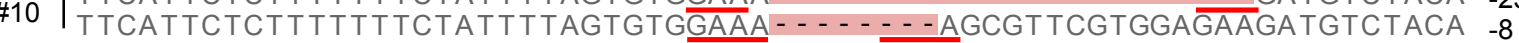

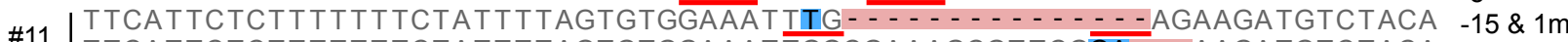

TTCATTCTCTTTTTTTCTATTTTAGTGTGGAAATGGCGAAGCGTTCG $\overline{\text { TA- }}$ - -AAGATGTCTACA $-3 \& 2 \mathrm{~m}$

\#12 ITCATTCTCTTTTTTTCTATTTTAGTGTGGAAAI - . - . - . - - - ICGTGGAGAAGATGTCTACA

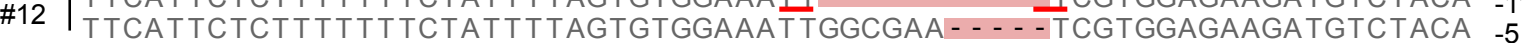

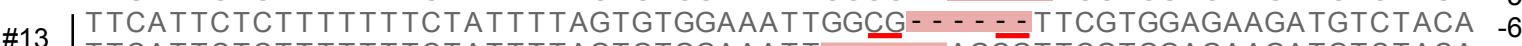

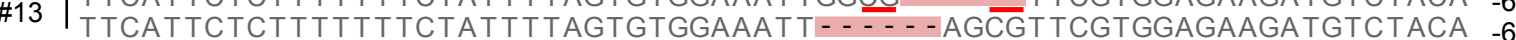

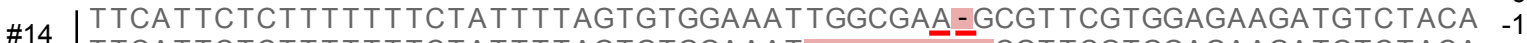

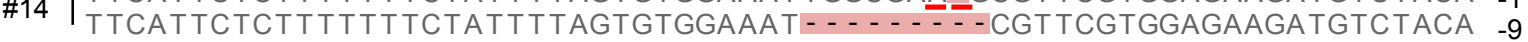

\#15 |TTCATTCTCTTTTTTTCTATTTTAGTGTGGAAATTGGCGAA - - - - - - - - - - - - GATGTCTACA

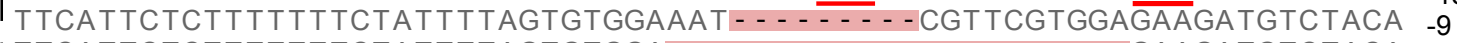

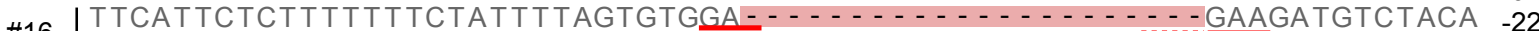

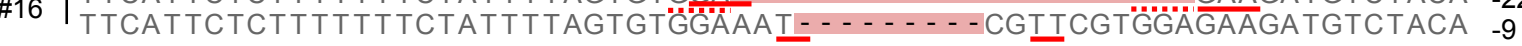

5'... TCCAGGGTGACAGCTGTGATACCCTGCTTCCCCTACGCCCGGCAAGATAAAAAGGACAAGGTAGGC ...3' Pros2 crRNA PAM

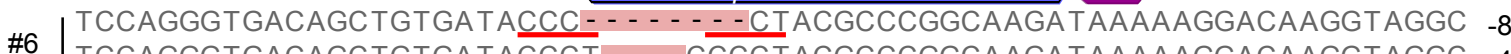

\#6 TCCAGgGTGACAGCTGTGATACCCT- - - -CCCCTACGCCCGGCAAGATAAAAAGGACAAGGTAGGC

5'... AGCAGGGTGACTGCAGTCATCCCGTGCTTCCCTTATGCCCGGCAGGATAAAAAGGATAAGAGCCGG ...3'

Pros 111 crRNA

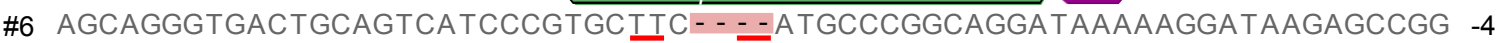

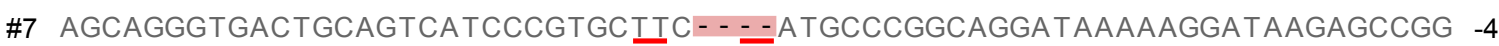

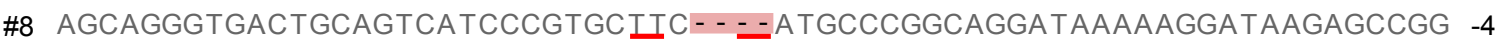

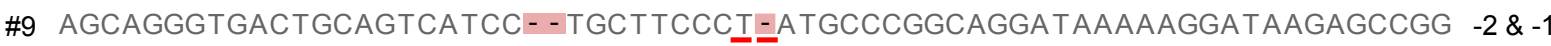

\#10 AGCAGGGTGACTGCAGTCATCCCGTGCTT\&CCCTTATGCCCGGCAGGATAAAAAGGATAAGAGCCGG +1

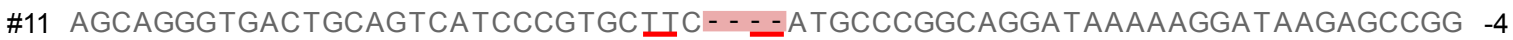

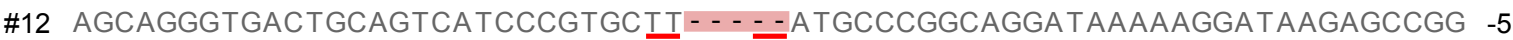

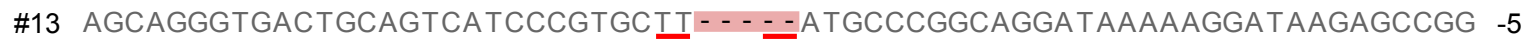

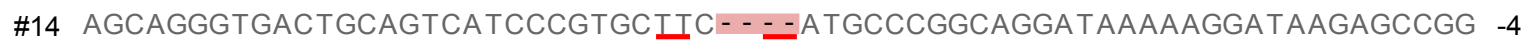

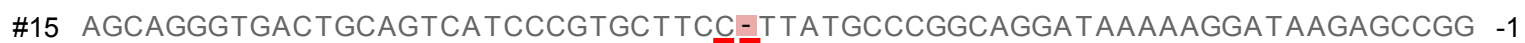

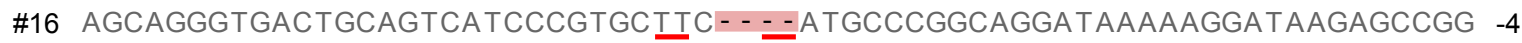

B

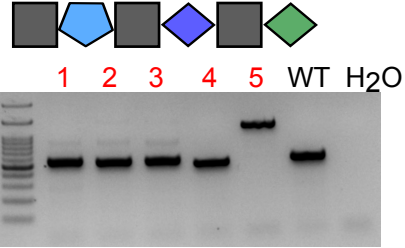

Direct repeat

miR10b

C

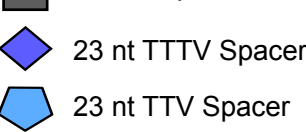

5'... TIGTAAOGTTGTCTATATATACOCTGTAGAACOGAATTGTGTGGTAOOCACATAGTCACAGATTOGATTCTAGGOGAATATATGGTOGATGCAAA ...3' miR10b precusor RNA

miR10b crRNA1 miR-10b-5p miR-miR-10b-3p

P1

TTGTAACGTTGCTATATATACOCTGTAGAACOGAAT-

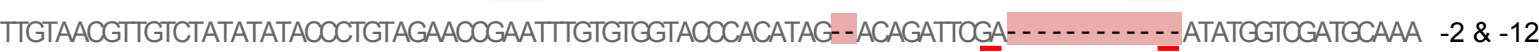

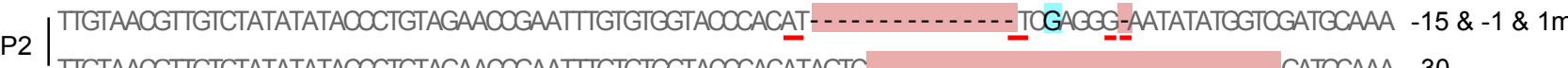

TाGTAACGTTGTCTATATATACOCTGTAGAACOGAATTGTGTGGTACCACATAGTC-

P3

TTGTAACGTTGTCTATATATACOCTGTAGAACOGAATTGTGTGGTACOCACATA- - - - - TTCGATTCTA- - - ATATATGGTCGATGCAAA

TTGTAAC-

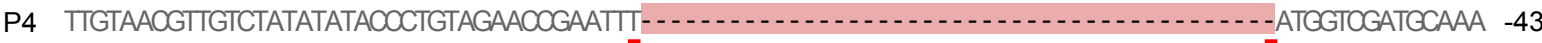

P5 TTGTAAOGTTGTCTATATATACOCTGTAGAACOGAATTTGTGTGGTACOCACAAATAATATCT 709 bp

TTOCACOOCTTGGTOGATCCAAA $-30 \&+709$ 


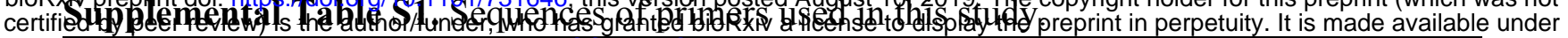

\section{Name}

T7 Top stand primer

T7 Mb3 Prps111 R

T7 Mb3 Mrvi1 cr(+TT) R

T7 Mb3 Saraf 2cr R

T7 Mb3 547509 R $\mathrm{R}$ crRNA1 \&3 R
T7 Mb3 prps fam new

\section{Sequence}

GAAATTAATACGACTCACTATAGGG TTCCCTTATGCCCGGCAGGAATCTACA AACAGTAGAAATTCCCTATAGTGAGT CGTATTAATTTC GTGCGCATGCAGAAGCTGACATCTAC AAACAGTAGAAATTTCTGGAGCAAGC ATCAGTGGATCTACAAACAGTAGAAA In vitro transcription of TTCCCTATAGTGAGTCGTATTAATTTC crRNA AGGGTGATACAGTGCCAGAAATCTAC AAACAGTAGAAATTCCCGCAAGAGTA TTCTGTCTATCTACAAACAGTAGAAAT In vitro transcription of TCCCTATAGTGAGTCGTATTAATTTC CCCAGGGTGATACAGTGCCAATCTAC AAACAGTAGAAATTCAAGAGTATTCT GTCTGAAAATCTACAAACAGTAGAAA In vitro transcription of T7 Mb3 Saraf 2ttcr R TTCCCTATAGTGAGTCGTATTAATTTC crRNA

TCTAGGGGAATATATGGTCGATGATCT ACAAACAGTAGAAATTTCTGTGACTAT GTGGGTACCACAATCTACAAACAGTA GAAATTCAGGGTATATATAGACAACG TTAATCTACAAACAGTAGAAATTCCCT In vitro transcription of ATAGTGAGTCGTATTAATTTC TGCTTCCCTTATGCCCGGCAGGAATCT ACAAACAGTAGAAATTTGCTTCCCCTA CGCCCGGCAAGAATCTACAAACAGTA GAAATTAACGCTTTCGCCAATTTCCAC ACATCTACAAACAGTAGAAATTCCCT ATAGTGAGTCGTATTAATTTC ATTACAGAAATGTCAATCACATTATCT ACAAACAGTAGAAATTAGAATGTGGC AATCATGCATAATATCTACAAACAGT AGAAATTGATGTACCAAGCATGTTTCC TGTCCCTATAGTGAGTCGTATTAATTT $\mathrm{C}$

TTCCTGCAGATGACGTCGGTCGCATCT ACAAACAGTAGAAATTTGCAGGAAGT AAAATGGCATATAATCTACAAACAGT AGAAATTCCCTATAGTGAGTCGTATTA In vitro transcription of ATTTC

CTCCCTCGCACCAGCCCCTGTGAATCT ACAAACAGTAGAAATTTGCGAGGGAG GACCATGGGTGGAATCTACAAACAGT

AGAAATTCCCTATAGTGAGTCGTATTA In vitro transcription of

T7 Mb3 Slit2 crRNA

Prps111 Ext F

ATTTC

GGAAGGGACAGTAACGGCTTT GATGCCATGAGTCAAGATGGCATAAA

Prps111 qPCR R CTC
crRNA

In vitro transcription of crRNA
In vitro transcription of crRNA
crRNA

Genotyping
Genotyping 


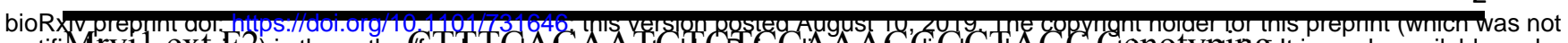

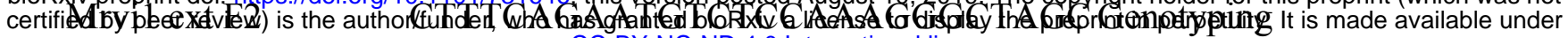

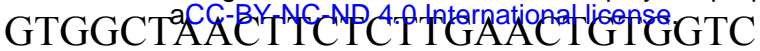

Mrvil ext R AC Genotyping CACGCTTTGTGTAGAACCATCAAAATG

Saraf ext F3

Saraf ext R5 TCTA

Genotyping GCATCACTGCATGGGAGGCC Genotyping

miR10b ext $\mathrm{F}$ miR $10 b$ ext $R$ TTAAGAAGAAGAAGGTCCTGGCTGCT CA Genotyping

AATACCCTAAAACCTGGCTCTCTGGC Genotyping CTTCTAGTATACAGGTTCCTTTAGGTG

Prps 1 ext F CTCTTTCTCTA Genotyping GCTGTCCGTTACAATGCCATTTCACAG

Prps1 ext R

Prps2 ext F2 TA Genotyping ACTCTGCTTTCCGATTAAGAGTGAAGT GTG Genotyping TTTGACTGCCTTTGAAACATACATTGC

Prps2 ext R2 CTG GAGACTCTTAGTTTCTAGATCTGGTTC

miR201 ext F2 CTT AAGAAGGACAGGGGTGGTAAAAGAG

miR547 ext R AAGTA AGATTGTGGTCAGGACAGAGATAGAG

miR509 ext $\mathrm{F}$

GAAA CACTGGTTGGGTGCAAATATCTACATC

miR509 ext $\mathrm{R}$

TGA

TTCATGTTTGACTTATAGGACCTCACG

Kenj10 geno $\mathrm{F}$

CTG

Genotyping TCGTCCATAGATCCTTGAGGTAGAGG

Kcnj10 geno R AAAC Genotyping Genotyping

BamHI opti linker F

AAGAAGGGATCCAGCGGTTCAGAGAC CCCAGGA Genotyping ACTGTGCTGGATATCTGCAGAATTCTC ATTTAACTTTGGTGAAGGTGTCCTGAC pcDNA3.1-Mb3Cas12a-

mSA GBS R $\mathrm{C}$ mSA construct pUC F TG

TGATGCGGTATTTTCTCCTTACGCATC pUC-Slit2-BamHI plasmid construct pUC-Slit2-BamHI

pUC R

ACATGTGAGCAAAAGGCCAGCAAAAG plasmid construct

Slit2 up GBS F CTGGCCTTTTGCTCACATGTTAAGTAC ATGTCACTGGATAAAGTCTTGAGC pUC-Slit2-BamHI GGCTGGATCCAAACTCACAGAACAAG pUC-Slit2-BamHI

Slit2 up GBS R CCACTGACA plasmid construct CTGTGAGTTTGGATCCAGCCCCTGTGA pUC-Slit2-BamHI

Slit2 down GBS F TAATTTTGATTGCCAGAATG plasmid construct AAGGAGAAAATACCGCATCAAAGAAT pUC-Slit2-BamHI

Slit2 down GBS R GTTCCTTGAAATTGTGCTTAAAGGC plasmid construct /5Biosg/TAAGTACATGTCACTGGATAA

Slit2 up bio $\mathrm{F}$ AGTCTTGAGC PCR for Biotin donor /5Biosg/AAGAATGTTCCTTGAAATTGTG PCR for Biotin donor CTTAAAGGC DNA 


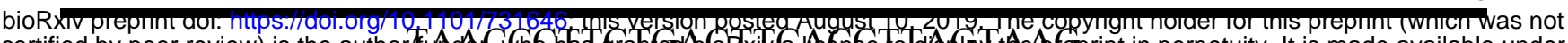

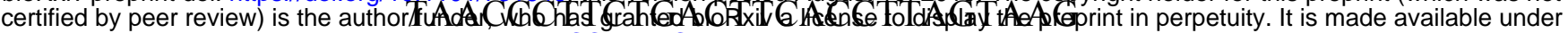

Slit2 ext F

CCA aCC-BY-NC-ND 4.0 International license.

Genotyping

Slit2 ext R

CTTGGCTGAAGGAATCTGAAGATAGG

U6 forward ACTC

Genotyping

GAGGGCCTATTTCCCATGATTCCTT PCR for Dnmt1 crRNA ACCGAGCAGGAGTGAGGATCTACAAA CAGTAGAAATTCGGTGTTTCGTCCTTT

U6 Mb3 Dnmt1 17nt CCAC

PCR for Dnmt1 crRNA

CACCGAGCAGGAGTGAGGATCTACAA

ACAGTAGAAATTCGGTGTTTCGTCCTT

U6 Mb3 Dnmt1 18nt

TCCAC

PCR for Dnmtl crRNA

TCACCGAGCAGGAGTGAGGATCTACA

AACAGTAGAAATTCGGTGTTTCGTCCT

U6 Mb3 Dnmt1 19nt TTCCAC

PCR for Dnmt1 crRNA

TTCACCGAGCAGGAGTGAGGATCTAC AAACAGTAGAAATTCGGTGTTTCGTCC

U6 Mb3 Dnmt1 20nt TTTCCAC

PCR for Dnmt1 crRNA

ATTCACCGAGCAGGAGTGAGGATCTA

CAAACAGTAGAAATTCGGTGTTTCGTC

U6 Mb3 Dnmt1 21nt CTTTCCAC

PCR for Dnmtl crRNA

AATTCACCGAGCAGGAGTGAGGATCT

ACAAACAGTAGAAATTCGGTGTTTCGT

U6 Mb3 Dnmt1 22nt CCTTTCCAC

PCR for Dnmt1 crRNA

AAATTCACCGAGCAGGAGTGAGGATC

TACAAACAGTAGAAATTCGGTGTTTCG

U6 Mb3 Dnmt1 23nt TCCTTTCCAC

PCR for Dnmt1 crRNA

Dnmt1 F2

CCGTTTTGGGCTCTGGGACTCA

Genotyping

AATCCAGAATGCACAAAGTACTGCAC

Dnmt1 R2

A

Genotyping 